\title{
WEIGHTED HARDY'S INEQUALITIES FOR NEGATIVE INDICES
}

\author{
DMitry V. Prokhorov
}

Abstract

In the paper we obtain a precise characterization of Hardy type inequalities with weights for the negative indices and the indices between 0 and 1 and establish a duality between these cases.

\section{Introduction}

The Hardy inequality with weights $u, v \geq 0$

$$
\left(\int_{0}^{+\infty} u(x)\left(\int_{0}^{x} f(y) v(y) d y\right)^{q} d x\right)^{\frac{1}{q}} \leq C\left(\int_{0}^{+\infty} f(x)^{p} d x\right)^{\frac{1}{p}}, \quad f \geq 0
$$

has been completely characterized for $p, q>0$ by G. Talenti [11], B. Muckenhoupt [7], J. S. Bradley [3], V. G. Maz'ja and A. Rozin [6, § 1.3], G. Sinnamon [9], G. Sinnamon and V. D. Stepanov [10] and some other authors (see the monographs $[\mathbf{8}]$ and $[\mathbf{5}]$ for details). An analogous problem for $p, q<1$ was studied by P. R. Beesack and H. P. Heinig [1] , where sufficient conditions and necessary conditions under some restrictions on the weight functions were given for the inequality

$$
\text { (1) }\left(\int_{0}^{+\infty} f(x)^{p} d x\right)^{\frac{1}{p}} \leq C\left(\int_{0}^{+\infty} u(x)\left(\int_{0}^{x} f(y) v(y) d y\right)^{q} d x\right)^{\frac{1}{q}}, \quad f \geq 0 .
$$

In the present paper we obtain a precise characterization of (1) for $p, q<0$ and $p, q \in(0,1)$. Moreover, we establish a duality between these cases.

The paper is organized as follows: Section 2 contains an explicit criterion of (1) for the cases $-\infty<q \leq p<0$ (Theorem 1) and $-\infty<p<$ $q<0$ (Theorem 2) and similar results with the dual operator. Theorem 3 of Section 3 proves that the Hardy type inequality with negative indices

2000 Mathematics Subject Classification. 26D15, 26D10.

Key words. Hardy's inequality, weight function, negative indices, duality.

The research was partially supported by the Russian Foundation for Basic Research (Project 03-01-00017) and by the Russian Academy of Science (Project 04-3- $-01-$ 049). 
of integration is equivalent to the same inequality with the dual operator for conjugate indices. As a consequence we are able to characterize (1) for $p, q \in(0,1)$ in the corollary of Section 4. Other propositions of Section 4 supplement the main results by a number of characterizations for similar inequalities.

Throughout this paper $A \lesssim B$ and $B \gtrsim A$ means that $A \leq c B$, where the constant $c$ depends only on $p, q$ and may be different in different places. If both $A \lesssim B$ and $A \gtrsim B$, then we write $A \approx B$. N stands for the set of positive integers, $\mathbb{Z}$ is the set of all integers and $(a, b)$ is a nonempty interval of the real line $(-\infty,+\infty)$. The symbol $p^{\prime}:=\frac{p}{p-1}$ denotes the conjugate number of $p, q^{\prime}:=\frac{q}{q-1}$ and the symbol $\square$ marks the end of proof of any statement. As usual we abbreviate "almost everywhere" by a.e. and "if and only if" by iff. Sometimes for simplicity we also use the notation $\int_{a}^{b} f:=\int_{a}^{b} f(x) d x$.

Let us mention one more thing before we start. A peculiarity of the inequality (1) caused by the negativity of the indices force us to work with measurable functions having their values in the extended semiaxis $[0,+\infty]$ equipped by arithmetic:

$$
\begin{aligned}
& 0+(+\infty)=a+(+\infty)=a \cdot(+\infty)=+\infty, \quad a \in(0,+\infty] ; \\
& 0 \cdot(+\infty)=0 ; \\
& (+\infty)^{\alpha}=0^{-\alpha}=+\infty, \quad(+\infty)^{-\alpha}=0^{\alpha}=0, \quad \alpha \in(0,+\infty) .
\end{aligned}
$$

Note also simple corollaries of the axioms (2) which we often use:

Let $\alpha, \beta \in(-\infty,+\infty) \backslash\{0\}$ and $a, b \in[0,+\infty]$. Then

1. $\left(a^{\alpha}\right)^{\beta}=a^{\alpha \beta}$.

2. $a^{\alpha} a^{\beta} \leq a^{\alpha+\beta}$; if $(a<+\infty$ and $\alpha+\beta>0)$ or $(a>0$ and $\alpha+\beta<0)$, then $a^{\alpha} a^{\beta}=a^{\alpha+\beta}$.

3. $a^{\alpha} b^{\alpha} \leq(a b)^{\alpha}$ with equality unless $\alpha<0$ and $\{a, b\}=\{0,+\infty\}$.

\section{The main results}

Denote by $\mathfrak{M}^{+}(a, b)$ the class of all measurable functions $f:(a, b) \rightarrow$ $[0,+\infty]$ and put

$$
\begin{aligned}
\left(I^{\times} f\right)(x) & :=\int_{a}^{x} f(y) d y, \\
\left(I_{\times} f\right)(x) & :=\int_{x}^{b} f(y) d y,
\end{aligned}
$$


Theorem 1. Let $-\infty<q \leq p<0, u, v \in \mathfrak{M}^{+}(a, b)$ and $I=I^{\times}$or $I=I_{\times}$. Then the following statement

(a) There exists a constant $C>0$ such that

(3) $\left(\int_{a}^{b} f(x)^{p} d x\right)^{\frac{1}{p}} \leq C\left(\int_{a}^{b} u(x)[(I(f v))(x)]^{q} d x\right)^{\frac{1}{q}}$

$$
\text { for all } f \in \mathfrak{M}^{+}(a, b)
$$

holds

is equivalent to $\mathcal{A}<+\infty$, where

$$
\mathcal{A}:=\sup _{a<t<b} \mathcal{A}(t):=\sup _{a<t<b}[(I u)(t)]^{-\frac{1}{q}}\left[\left(I v^{p^{\prime}}\right)(t)\right]^{-\frac{1}{p^{\prime}}} .
$$

Moreover, $\mathcal{A} \approx C$ for the least possible constant $C$ in $(3)$.

Proof: Let $I=I^{\times}$(the case $I=I_{\times}$can be proved analogously). At first we note that finiteness of the constant $\mathcal{A}$ is equivalent to the condition $\left(a_{1}\right) \int_{a}^{a^{*}} u=0, \int_{a^{*}}^{t} u<+\infty$ for all $t \in\left(a^{*}, b^{*}\right), A<+\infty$ and $\int_{a^{*}}^{b^{*}} u=$ $+\infty$ implies $\int_{a^{*}}^{b^{*}} v^{p^{\prime}}=+\infty$, where $a^{*}:=\sup \left\{t \in[a, b) \mid \int_{a}^{t} v^{p^{\prime}}=0\right\}$, $b^{*}:=\sup \left\{t \in[a, b) \mid \int_{a}^{t} v^{p^{\prime}}<+\infty\right\}$ and

$$
A:=\sup _{a^{*}<t<b^{*}} A(t):=\sup _{a^{*}<t<b^{*}}\left(\int_{a^{*}}^{t} u\right)^{-\frac{1}{q}}\left(\int_{a^{*}}^{t} v^{p^{\prime}}\right)^{-\frac{1}{p^{\prime}}} .
$$

Moreover, $\mathcal{A}=A$. If $\left(a_{1}\right)$ holds then for $t \in\left(a, a^{*}\right]$ we have $\left(\int_{a}^{t} u\right)^{-\frac{1}{q}}=0$ and for $t \in\left(b^{*}, b\right)$ we have $\left(\int_{a}^{t} v^{p^{\prime}}\right)^{-\frac{1}{p^{\prime}}}=0$. For $t \in\left(a^{*}, b^{*}\right]$ we have $\mathcal{A}(t)=A(t) \leq A$. Putting these together shows that $\mathcal{A} \leq A<+\infty$. Conversely, if $\mathcal{A}<+\infty$ then $\mathcal{A}\left(a^{*}\right)<+\infty$ and $\int_{a}^{a^{*}} v^{p^{\prime}}=0$ so $\int_{a}^{a^{*}} u=0$. The condition that $\int_{a^{*}}^{t} u<+\infty$ for $t \in\left(a^{*}, b^{*}\right)$ is evident because $\int_{a}^{t} v^{p^{\prime}} \in(0,+\infty)$ there. If $\int_{a^{*}}^{b^{*}} u=+\infty$ then we must have $\left(\int_{a^{*}}^{b^{*}} v^{p^{\prime}}\right)^{-\frac{1}{p^{\prime}}}=0$ so $\int_{a^{*}}^{b^{*}} v^{p^{\prime}}=+\infty$.

Finally, because $\int_{a}^{a^{*}} v^{p^{\prime}}=0$ we obtain $A \leq \mathcal{A}<+\infty$ and we have completed $\left(a_{1}\right)$. 
Let $(a)$ hold. Assume that $a^{*}<b^{*}$. Fix an arbitrary $t \in\left(a^{*}, b^{*}\right)$. Substituting $f_{t}(x)=+\infty \chi_{(t, b)}(x)+v(x)^{p^{\prime}-1} \chi_{(a, t)}(x)$ into (3), we obtain

$$
\begin{aligned}
0<\left(\int_{a}^{t} v^{p^{\prime}}\right)^{\frac{1}{p}} & \leq C\left(\int_{a}^{t} u(x)\left(\int_{a}^{x} v^{p^{\prime}}\right)^{q} d x\right)^{\frac{1}{q}} \\
& =C\left(\int_{a}^{a^{*}} u(x)\left(\int_{a}^{x} v^{p^{\prime}}\right)^{q} d x+\int_{a^{*}}^{t} u(x)\left(\int_{a}^{x} v^{p^{\prime}}\right)^{q} d x\right)^{\frac{1}{q}},
\end{aligned}
$$

since $v<+\infty$ a.e. on $\left(a, b^{*}\right)$. It implies

$$
\int_{a}^{a^{*}} u(x)\left(\int_{a}^{x} v^{p^{\prime}}\right)^{q} d x<+\infty \text { and } \int_{a^{*}}^{t} u(x)\left(\int_{a}^{x} v^{p^{\prime}}\right)^{q} d x<+\infty,
$$

otherwise we have a contradiction $0<0$. Since for $x \in\left(a, a^{*}\right)$ it holds that $\int_{a}^{x} v^{p^{\prime}}=0$, we have $\int_{a}^{a^{*}} u=0$ and

$$
\left(\int_{a^{*}}^{t} v^{p^{\prime}}\right)^{\frac{1}{p}} \leq C\left(\int_{a^{*}}^{t} u\right)^{\frac{1}{q}}\left(\int_{a^{*}}^{t} v^{p^{\prime}}\right),
$$

because $\int_{a^{*}}^{t} v^{p^{\prime}} \in(0,+\infty)$. If $\int_{a^{*}}^{t} u=+\infty$, then (4) implies that $\int_{a^{*}}^{t} v^{p^{\prime}}=$ $+\infty$ which contradicts with the definition of $b^{*}$. If $\int_{a^{*}}^{t} u=0$, then $A(t)=0$. If $\int_{a^{*}}^{t} u \in(0,+\infty)$, then it follows from (4) that $A(t) \leq C$.

Analogously, using the function $f_{t}$ with $t=b^{*}$ we obtain (4) with $t=$ $b^{*}$ and this inequality implies that $\int_{a^{*}}^{b^{*}} v^{p^{\prime}}=+\infty$ in the case $\int_{a^{*}}^{b^{*}} u=+\infty$.

Now if $a^{*}=b^{*}=b$, then $\int_{a}^{b} v=0$ and by putting $f=+\infty$ into (3) we get

$$
+\infty \leq C\left(\int_{a}^{b} u(x) \cdot(+\infty) d x\right)^{\frac{1}{q}}
$$

Thus, $\int_{a}^{b} u=0$ and all the conditions of $\left(a_{1}\right)$ hold, as well as, in the case $a^{*}=b^{*}=a$.

Let $\left(a_{1}\right)$ hold. Since $\int_{a}^{a^{*}} u=\int_{a}^{a^{*}} v^{p^{\prime}}=0$, it yields that (3) is equivalent to the inequality

$$
\begin{array}{r}
\int_{a^{*}}^{b} u(x)\left(\int_{a^{*}}^{x} v(y) g(y)^{\frac{1}{q}} d y\right)^{q} d x \leq C^{-q}\left(\int_{a}^{b} g^{\frac{p}{q}}\right)^{\frac{q}{p}} \\
\text { for all } g \in \mathfrak{M}^{+}(a, b) .
\end{array}
$$

If $a^{*}=b^{*}=b$, then (5) holds. Let $a^{*}<b^{*}$ or $a^{*}=b^{*}=a$. Fix an arbitrary non negative measurable function $g$ on $(a, b)$. If $\int_{a}^{b} g^{\frac{p}{q}}=+\infty$, 
HaRdy's Inequalities for Negative Indices

427

then we have (5). Now let $\int_{a}^{b} g^{\frac{p}{q}}<+\infty$. If $b^{*}<b$, then, by Hölder's inequality with exponents $p$ and $p^{\prime}$, we find that for any $x \in\left(b^{*}, b\right)$

$$
\int_{a^{*}}^{x} v(y) g(y)^{\frac{1}{q}} d y \geq\left(\int_{a^{*}}^{x} v^{p^{\prime}}\right)^{\frac{1}{p^{\prime}}}\left(\int_{a^{*}}^{x} g^{\frac{p}{q}}\right)^{\frac{1}{p}}=+\infty .
$$

Thus,

(6) $J_{1}:=\int_{a^{*}}^{b} u(x)\left[\int_{a^{*}}^{x} v(y) g(y)^{\frac{1}{q}} d y\right]^{q} d x=\int_{a^{*}}^{b^{*}} u(x)\left[\int_{a^{*}}^{x} v(y) g(y)^{\frac{1}{q}} d y\right]^{q} d x$.

In particular, if $a^{*}=b^{*}=a$, then (5) holds.

Now let $a^{*}<b^{*}$. Put

$$
N:= \begin{cases}\inf \left\{k \in \mathbb{Z} \mid k \geq \log _{2}\left(\int_{a^{*}}^{b^{*}} v^{p^{\prime}}\right)\right\}, & \text { if } \int_{a^{*}}^{b^{*}} v^{p^{\prime}}<+\infty, \\ +\infty, & \text { otherwise, }\end{cases}
$$

and construct the sequence $\left\{a_{k}\right\}_{k \leq N}$ by the relations: $\int_{a^{*}}^{a_{k}} v^{p^{\prime}}=2^{k}$, $k<N ; a_{N}=b^{*}$. Then it follows from (6) that

$$
J_{1}=\sum_{k<N} \int_{a_{k}}^{a_{k+1}} u(x)\left(\int_{a^{*}}^{x} v(y) g(y)^{\frac{1}{q}} d y\right)^{q} d x
$$

Note that $\int_{a_{k}}^{a_{k+1}} u<+\infty$ for all $k<N$ since $\int_{a^{*}}^{t} u<+\infty$ for all $t \in$ $\left(a^{*}, b^{*}\right)$ and $\int_{a^{*}}^{b^{*}} u=+\infty$ implies that $\int_{a^{*}}^{b^{*}} v^{p^{\prime}}=+\infty$.

By applying the Hölder inequality with exponents $p^{\prime}$ and $p$, we have for all $x \in\left(a^{*}, b^{*}\right)$

$$
\begin{aligned}
\int_{a^{*}}^{x} v(y) g(y)^{\frac{1}{q}} d y & =\int_{a^{*}}^{x} v(y) g(y)^{\frac{1}{q}}\left[\int_{a^{*}}^{y} v^{p^{\prime}}\right]^{\frac{1}{p p^{\prime}}-\frac{1}{p p^{\prime}}} d y \\
& \geq\left(\int_{a^{*}}^{x} v(y)^{p^{\prime}}\left[\int_{a^{*}}^{y} v^{p^{\prime}}\right]^{-\frac{1}{p}} d y\right)^{\frac{1}{p^{\prime}}}\left(\int_{a^{*}}^{x} g(y)^{\frac{p}{q}}\left[\int_{a^{*}}^{y} v^{p^{\prime}}\right]^{\frac{1}{p^{\prime}}} d y\right)^{\frac{1}{p}} \\
& =\left(p^{\prime}\right)^{\frac{1}{p^{\prime}}}\left[\int_{a^{*}}^{x} v^{p^{\prime}}\right]^{\frac{1}{p^{\prime 2}}}\left(\int_{a^{*}}^{x} g(y)^{\frac{p}{q}}\left[\int_{a^{*}}^{y} v^{p^{\prime}}\right]^{\frac{1}{p^{\prime}}} d y\right)^{\frac{1}{p}} .
\end{aligned}
$$


Using this relation and (7) we estimate the left part of (5) as follows:

$$
\begin{aligned}
J_{1} & \lesssim \sum_{k<N}\left(\int_{a_{k}}^{a_{k+1}} u\right)\left[\int_{a^{*}}^{a_{k}} v^{p^{\prime}}\right]^{\frac{q}{p^{\prime 2}}}\left(\int_{a^{*}}^{a_{k+1}} g(y)^{\frac{p}{q}}\left[\int_{a^{*}}^{y} v^{p^{\prime}}\right]^{\frac{1}{p^{\prime}}} d y\right)^{\frac{q}{p}} \\
& \leq \sum_{k<N}\left(\int_{a_{k}}^{a_{k+1}} u\right)\left(2^{k}\right)^{\frac{q}{p^{\prime 2}}}\left(\sum_{j \leq k}\left[\int_{a_{j}}^{a_{j+1}} g^{\frac{p}{q}}\right]\left(2^{j+1}\right)^{\frac{1}{p^{\prime}}}\right)^{\frac{q}{p}} .
\end{aligned}
$$

Moreover, by applying Minkowski's inequality we finally obtain that

$$
\begin{aligned}
J_{1} & \lesssim\left(\sum_{j<N}\left[\int_{a_{j}}^{a_{j+1}} g^{\frac{p}{q}}\right]\left(2^{j+1}\right)^{\frac{1}{p^{\prime}}}\left(\sum_{j \leq k<N}\left[\int_{a_{k}}^{a_{k+1}} u\right]\left(2^{k}\right)^{\frac{q}{p^{\prime 2}}}\right)^{\frac{p}{q}}\right)^{\frac{q}{p}} \\
& \lesssim A^{-q}\left(\sum_{j<N}\left[\int_{a_{j}}^{a_{j+1}} g^{\frac{p}{q}}\right]\left(2^{j}\right)^{\frac{1}{p^{\prime}}}\left[\sum_{j \leq k<N}\left(2^{k}\right)^{-\frac{q}{p^{\prime} p}}\right]^{\frac{p}{q}}\right)^{\frac{q}{p}} \\
& \lesssim A^{-q}\left[\int_{a}^{b} g^{\frac{p}{q}}\right]^{\frac{q}{p}} .
\end{aligned}
$$

The proof is complete.

Theorem 2. Let $-\infty<p<q<0, \frac{1}{r}:=\frac{1}{q}-\frac{1}{p}, u, v \in \mathfrak{M}^{+}(a, b)$ and $I=I^{\times}$or $I=I_{\times}$. Then $(a)$ is equivalent to $\mathcal{B}<+\infty$, where

$$
\mathcal{B}:=\left(\int_{a}^{b}[(I u)(x)]^{\frac{r}{p}}\left[\left(I v^{p^{\prime}}\right)(x)\right]^{\frac{r}{p^{\prime}}} u(x) d x\right)^{-\frac{1}{r}}
$$

Moreover, $\mathcal{B} \approx C$ for the least possible constant $C$ in (3).

Proof: As in Theorem 1 we only consider the case $I=I^{\times}$and note that finiteness of $\mathcal{B}$ is equivalent to the condition

$\left(a_{2}\right) \int_{a}^{a^{*}} u=0, \int_{a^{*}}^{t} u<+\infty$ for all $t \in\left(a^{*}, b^{*}\right), B<+\infty$ and $\int_{a^{*}}^{b^{*}} u=+\infty$ implies $\int_{a^{*}}^{b^{*}} v^{p^{\prime}}=+\infty$, where $a^{*}, b^{*}$ be the same as in Theorem 1 and

$$
B:=\left(\int_{a^{*}}^{b^{*}}\left[\int_{a^{*}}^{x} u\right]^{\frac{r}{p}}\left[\int_{a^{*}}^{x} v^{p^{\prime}}\right]^{\frac{r}{p^{\prime}}} u(x) d x\right)^{-\frac{1}{r}}
$$


Moreover, $\mathcal{B}=B$. If $\left(a_{2}\right)$ holds, then $\mathcal{B}=B$, that is $\mathcal{B}<+\infty$. Conversely, let $\mathcal{B}<+\infty$. Since $\mathcal{B} \geq\left(\frac{q}{r}\right)^{-\frac{1}{r}} \mathcal{A}$, all conditions of $\left(a_{2}\right)$, except $B<+\infty$, follow from finiteness of $\mathcal{A}$ in the same way as in the proof of Theorem 1 . It implies the equality $B=\mathcal{B}$ and $\left(a_{2}\right)$ follows.

The case $a^{*}=b^{*}$ can be proved analogously with the proof of Theorem 1. Therefore, we assume that $a^{*}<b^{*}$.

Let $(a)$ hold. All conditions, except $B<+\infty$, follow in the same way as in the proof of Theorem 1 . We only need to show that $B$ is finite. If $\int_{a^{*}}^{b^{*}} u=0$, then $B=0<+\infty$. Let $\int_{a^{*}}^{b^{*}} u>0$. Then there exist numbers $t_{1}$ and $t_{2}$ such that $a^{*}<t_{1}<t_{2}<b^{*}$ and $\int_{t_{1}}^{t_{2}} u>0$. Denote $\tilde{u}:=u \chi_{\left(t_{1}, t_{2}\right)}$ and let $\tilde{B}$ be similar to $B$ with $\tilde{u}$ instead of $u$. Then

$$
\begin{aligned}
\tilde{B} & =\left[\int_{t_{1}}^{t_{2}}\left[\int_{t_{1}}^{x} u\right]^{\frac{r}{p}}\left[\int_{a^{*}}^{x} v^{p^{\prime}}\right]^{\frac{r}{p^{\prime}}} \tilde{u}(x) d x\right]^{-\frac{1}{r}} \\
& \leq\left[\int_{a^{*}}^{t_{1}} v^{p^{\prime}}\right]^{-\frac{1}{p^{\prime}}}\left[\int_{t_{1}}^{t_{2}} u\right]^{-\frac{1}{q}}<+\infty
\end{aligned}
$$

$\tilde{B}>0$ and the inequality $(3)$ holds with $\tilde{u}$ instead of $u$ :

$$
\left(\int_{a}^{b} f(x)^{p} d x\right)^{\frac{1}{p}} \leq C\left(\int_{a}^{b} \tilde{u}(x)\left(\int_{a}^{x} f v\right)^{q} d x\right)^{\frac{1}{q}} \text { for all } f \in \mathfrak{M}^{+}(a, b) \text {. }
$$

The last inequality is equivalent to

(8) $\int_{a^{*}}^{b^{*}} \tilde{u}(x)\left(\int_{a^{*}}^{x} v(y) g(y)^{\frac{1}{q}} d y\right)^{q} d x \leq C^{-q}\left(\int_{a}^{b} g^{\frac{p}{q}}\right)^{\frac{q}{p}}$

for all $g \in \mathfrak{M}^{+}(a, b)$.

Put

$$
g(y)^{\frac{p}{q}}:=v(y)^{p^{\prime}}\left(\int_{y}^{b^{*}} \tilde{u}(z)\left(\int_{a^{*}}^{z} v^{p^{\prime}}\right)^{q-1} d z\right)^{\frac{r}{q}} \chi_{\left(a^{*}, b^{*}\right)}(y) .
$$


Then

$$
\int_{a}^{b} g^{\frac{p}{q}}=\int_{a^{*}}^{b^{*}} v(y)^{p^{\prime}}\left(\int_{y}^{b^{*}} \tilde{u}(z)\left(\int_{a^{*}}^{z} v^{p^{\prime}}\right)^{q-1} d z\right)^{\frac{r}{q}} d y=: J_{2}
$$

and since $v<+\infty$ a.e. on $\left(a^{*}, b^{*}\right)$ and $\int_{y}^{b^{*}} \tilde{u}(z)\left(\int_{a^{*}}^{z} v^{p^{\prime}}\right)^{q-1} d z<+\infty$ for all $y \in\left(a^{*}, b^{*}\right)$ we have

$$
\begin{aligned}
& \int_{a^{*}}^{b^{*}} \tilde{u}(x)\left(\int_{a^{*}}^{x} v(y) g(y)^{\frac{1}{q}} d y\right)^{q} d x \\
& \geq \int_{a^{*}}^{b^{*}} \tilde{u}(x)\left[\int_{x}^{b^{*}} \tilde{u}(z)\left[\int_{a^{*}}^{z} v^{p^{\prime}}\right]^{q-1} d z\right]^{\frac{r}{p}}\left[\int_{a^{*}}^{x} v^{p^{p^{\prime}}}\right]^{q-1}\left\{\int_{a^{*}}^{x} v(y)^{p^{\prime}} d y\right\} d x \\
& =\int_{a^{*}}^{b^{*}} v(y)^{p^{\prime}} \int_{y}^{b^{*}} \tilde{u}(x)\left[\int_{a^{*}}^{x} v^{p^{\prime}}\right]^{q-1}\left[\int_{x}^{b^{*}} \tilde{u}(z)\left[\int_{a^{*}}^{z} v^{p^{\prime}}\right]^{q-1} d z\right]^{\frac{r}{p}} d x d y=\frac{q}{r} J_{2},
\end{aligned}
$$

so that, by (8), $\frac{q}{r} J_{2} \leq C^{-q} J_{2}^{\frac{q}{p}}$.

Let $\left\{a_{k}\right\}_{k \leq N}$ be the same sequence as in the proof of Theorem 1 . Recall that $\int_{a_{k}}^{a_{k+1}} \tilde{u}<+\infty$ for all $k<N$ and we have

$$
\begin{aligned}
J_{2} & \leq \sum_{k<N}\left[\int_{a_{k}}^{a_{k+1}} v^{p^{\prime}}\right]\left(\int_{a_{k}}^{b^{*}} \tilde{u}(z)\left[\int_{a^{*}}^{z} v^{p^{\prime}}\right]^{q-1} d z\right)^{\frac{r}{q}} \\
& \lesssim \sum_{k<N} 2^{k}\left(\sum_{k \leq j<N}\left[\int_{a_{j}}^{a_{j+1}} \tilde{u}\right]\left(2^{j}\right)^{q-1}\right)^{\frac{r}{q}} .
\end{aligned}
$$

Putting $\beta_{j}^{\frac{r}{q}}:=\left(2^{j}\right)^{\frac{r}{p^{\prime}}}\left(\int_{a_{j}}^{a_{j+1}} \tilde{u}\right)^{\frac{r}{q}}$, we find that

$$
J_{2} \lesssim \sum_{k<N} 2^{k}\left(\sum_{k \leq j<N} \beta_{j}\left(2^{j}\right)^{-\frac{q}{r}}\right)^{\frac{r}{q}} \lesssim \sum_{k<N} \beta_{k}^{\frac{r}{q}}
$$

by the discrete Hardy inequality (see e.g. [2]) and since for any fixed $n \in \mathbb{Z}$

$$
\left(\sum_{k \leq n} 2^{k}\right)^{\frac{q}{r}}\left(\sum_{j \geq n}\left[\left(2^{j}\right)^{-\frac{q}{r}}\right]^{\frac{p}{q}}\right)^{\frac{q}{p}} \lesssim 1
$$


holds. Conversely,

$$
\begin{aligned}
J_{2} & \geq \sum_{k<N}\left[\int_{a_{k-1}}^{a_{k}} v^{p^{\prime}}\right]\left(\int_{a_{k}}^{b^{*}} \tilde{u}(z)\left[\int_{a^{*}}^{z} v^{p^{\prime}}\right]^{q-1} d z\right)^{\frac{r}{q}} \\
& \gtrsim \sum_{k<N} 2^{k}\left[\int_{a_{k}}^{a_{k+1}} \tilde{u}\right]^{\frac{r}{q}}\left(2^{k}\right)^{(q-1) \frac{r}{q}}=\sum_{k<N} \beta_{k}^{\frac{r}{q}} .
\end{aligned}
$$

Analogously,

$$
\begin{aligned}
\tilde{B}^{-r} & \lesssim \sum_{k<N}\left[\int_{a_{k}}^{a_{k+1}} \tilde{u}\right]\left[\int_{a^{*}}^{a_{k+1}} \tilde{u}\right]^{\frac{r}{p}}\left(2^{k}\right)^{\frac{r}{p^{\prime}}} \\
& \leq \sum_{k<N}\left(2^{k}\right)^{\frac{r}{p^{\prime}}}\left[\sum_{j \leq k} \int_{a_{j}}^{a_{j+1}} \tilde{u}\right]^{\frac{r}{q}} \\
& =\sum_{k<N}\left(2^{k}\right)^{\frac{r}{p^{\prime}}}\left[\sum_{j \leq k} \beta_{j}\left(2^{j}\right)^{-\frac{q}{p^{\prime}}}\right]^{\frac{r}{q}} \\
& \lesssim \sum_{k<N} \beta_{k}^{\frac{r}{q}}
\end{aligned}
$$

and

$$
\begin{aligned}
\tilde{B}^{-r} & \gtrsim \sum_{k<N} \int_{a_{k}}^{a_{k+1}} \tilde{u}(x)\left(\int_{a^{*}}^{x} \tilde{u}\right)^{\frac{r}{p}} d x\left(2^{k}\right)^{\frac{r}{p^{\prime}}} \\
& \geq \sum_{k<N}\left(2^{k}\right)^{\frac{r}{p^{\prime}}} \int_{a_{k}}^{a_{k+1}} \tilde{u}(x)\left(\int_{a_{k}}^{x} \tilde{u}\right)^{\frac{r}{p}} d x=\frac{q}{r} \sum_{k<N} \beta_{k}^{\frac{r}{q}} .
\end{aligned}
$$

Thus,

$$
\tilde{B}^{-r} \lesssim J_{2} \leq C^{-q} J_{2}^{\frac{q}{p}} \lesssim C^{-q}\left(\tilde{B}^{-r}\right)^{\frac{q}{p}},
$$

that is $\tilde{B} \lesssim C$, since $\tilde{B} \in(0,+\infty)$. By now letting $t_{1} \rightarrow a^{*}$ and $t_{2} \rightarrow b^{*}$, we conclude that $B<+\infty$ and the proof of the implication $(a) \Rightarrow\left(a_{2}\right)$ is complete.

Conversely, assume that $\left(a_{2}\right)$ holds. Fix any non-negative measurable function $g$ on $(a, b)$. By arguing similar as in the proof that $\left(a_{1}\right)$ 
implies $(a)$ in Theorem 1, we find that

$$
\begin{aligned}
& \int_{a}^{b} u(x)\left(\int_{a}^{x} v(y) g(y)^{\frac{1}{q}} d y\right)^{q} d x \\
& \lesssim \sum_{k<N}\left(2^{k}\right)^{\frac{q}{p^{\prime 2}}}\left[\int_{a_{k}}^{a_{k+1}} u\right]\left(\sum_{j \leq k}\left(2^{j}\right)^{\frac{1}{p^{\prime}}} \int_{a_{j}}^{a_{j+1}} g^{\frac{p}{q}}\right)^{\frac{q}{p}} \\
& \quad=\sum_{k<N}\left\{\left(2^{k}\right)^{\frac{q}{p^{\prime}}} \int_{a_{k}}^{a_{k+1}} u\right\}\left\{\left(2^{k}\right)^{\frac{q\left(1-p^{\prime}\right)}{p^{\prime 2}}}\left(\sum_{j \leq k}\left(2^{j}\right)^{\frac{1}{p^{\prime}}} \int_{a_{j}}^{a_{j+1}} g^{\frac{p}{q}}\right)^{\frac{q}{p}}\right\}
\end{aligned}
$$

and, by applying Hölder's inequality for sums with exponents $\frac{r}{q}$ and $\frac{p}{q}$, we have

$$
\begin{aligned}
& \leq\left(\sum_{k<N}\left(2^{k}\right)^{\frac{r}{p^{\prime}}}\left[\int_{a_{k}}^{a_{k+1}} u\right]^{\frac{r}{q}}\right)^{\frac{q}{r}}\left(\sum_{k<N}\left(2^{k}\right)^{-\frac{1}{p^{\prime}}} \sum_{j \leq k}\left(2^{j}\right)^{\frac{1}{p^{\prime}}} \int_{a_{j}}^{a_{j+1}} g^{\frac{p}{q}}\right)^{\frac{q}{p}} \\
& \lesssim B^{-q}\left(\sum_{j<N}\left[\int_{a_{j}}^{a_{j+1}} g^{\frac{p}{q}}\right]\left(2^{j}\right)^{\frac{1}{p^{\prime}}} \sum_{j \leq k<N}\left(2^{k}\right)^{-\frac{1}{p^{\prime}}}\right)^{\frac{q}{p}} \\
& \lesssim B^{-q}\left(\int_{a}^{b} g^{\frac{p}{q}}\right)^{\frac{q}{p}} .
\end{aligned}
$$

This end the proof.

\section{Principle of duality}

The following statement supplements a well-known result in [4] (see Theorem 234).

Lemma. Let $p \in(-\infty, 0) \cup(0,1)$ and $f \in \mathfrak{M}^{+}(a, b)$. Then

(9) $\quad \int_{a}^{b} f(x) g(x) d x \geq C\left(\int_{a}^{b} g(x)^{p^{\prime}} d x\right)^{\frac{1}{p^{\prime}}}$ for all $g \in \mathfrak{M}^{+}(a, b)$

holds iff

$$
\left(\int_{a}^{b} f(x)^{p} d x\right)^{\frac{1}{p}} \geq C
$$


Proof: Sufficiency. Follows from the Hölder inequality.

Necessity. The result for the case $p \in(0,1)$ is well known (see e.g. [4, Theorem 234]). Let $p \in(-\infty, 0)$ and $C>0$. Denote $E:=\{x \in(a, b) \mid$ $f(x)=0\}$ and insert $g=\chi_{E}$ into (9). Then we obtain that mes $E=0$. Fix a function $f_{0} \in \mathfrak{M}^{+}(a, b)$ such that $f_{0}(x) \in(0, \infty), x \in(a, b)$ and $\int_{a}^{b} f_{0}(x)^{p}<+\infty$ and put

$$
\begin{aligned}
& f_{n}=\max \left\{n^{-1} f_{0}, f\right\}, \quad n \in \mathbb{N} . \\
& g_{n}=f_{n}^{p-1},
\end{aligned}
$$

Then $\int_{a}^{b} f_{n}(x)^{p}<+\infty$ and by $(9)$

$$
\begin{aligned}
\int_{a}^{b} f_{n}(x)^{p} d x & =\int_{a}^{b} f_{n}(x) g_{n}(x) d x \geq \int_{a}^{b} f(x) g_{n}(x) d x \\
& \geq C\left(\int_{a}^{b} g_{n}(x)^{p^{\prime}} d x\right)^{\frac{1}{p^{\prime}}}=C\left(\int_{a}^{b} f_{n}(x)^{p} d x\right)^{\frac{1}{p^{\prime}}}
\end{aligned}
$$

for all $n \in \mathbb{N}$. Hence,

$$
\left(\int_{a}^{b} f_{n}(x)^{p} d x\right)^{\frac{1}{p}} \geq C \text { for all } n \in \mathbb{N} .
$$

Since $f_{n}(x)^{p} \uparrow f(x)^{p}, n \rightarrow \infty$, for every $x \in(a, b)$ the last inequality implies (10) by the Monotone Convergence Theorem.

Our principle of duality reads:

Theorem 3. Let $-\infty<p, q<0,0<C<+\infty$, $\mathrm{k}$ be a non-negative measurable function on $(a, b) \times(a, b)$ and

$$
\begin{aligned}
& (T f)(x):=\int_{a}^{b} \mathrm{k}(x, y) f(y) d y, \quad x \in(a, b) . \\
& \left(T^{*} g\right)(x):=\int_{a}^{b} \mathrm{k}(y, x) g(y) d y,
\end{aligned}
$$

Then the inequality

(11) $\left(\int_{a}^{b} f(x)^{p} d x\right)^{\frac{1}{p}} \leq C\left(\int_{a}^{b}[(T f)(x)]^{q} d x\right)^{\frac{1}{q}}$ for all $f \in \mathfrak{M}^{+}(a, b)$

holds iff the inequality

(12) $\left(\int_{a}^{b} g(x)^{q^{\prime}} d x\right)^{\frac{1}{q^{\prime}}} \leq C\left(\int_{a}^{b}\left[\left(T^{*} g\right)(x)\right]^{p^{\prime}} d x\right)^{\frac{1}{p^{\prime}}}$ for all $g \in \mathfrak{M}^{+}(a, b)$

holds. In particular, it follows that the least possible constants in (11) and (12) are equal. 
434

D. V. Prokhorov

Proof: Both (11) and (12) holds eff the inequality

$$
\left(\int_{a}^{b} f(x)^{p} d x\right)^{\frac{1}{p}}\left(\int_{a}^{b} g(x)^{q^{\prime}} d x\right)^{\frac{1}{q^{\prime}}} \leq C \int_{a}^{b} g(T f)=C \int_{a}^{b} f\left(T^{*} g\right)
$$

holds for all $f, g \in \mathfrak{M}^{+}(a, b)$ by the lemma and the proof follows.

4. Further results

In this section we show that in general the Hardy type inequalities with negative indices essentially depend on the location of the weight functions and the order of operations. By combining the propositions of this section and the theorems of the previous sections, it is easy to get suitable characterization of a number of closely related inequalities considered in this section.

Throughout this section $C \in(0,+\infty)$ and $u, v \in \mathfrak{M}^{+}(a, b)$.

Example 1. Let $u=\chi_{(0,1)}$ and $v=+\infty \chi_{(1,+\infty)}+\chi_{(0,1)}$. Then the inequality

$$
\left(\int_{0}^{+\infty}\left[f(y) v(y)^{-1}\right]^{-1} d y\right)^{-1} \leq C\left(\int_{0}^{+\infty} u(x)\left(\int_{0}^{x} f(y) d y\right)^{-1} d x\right)^{-1}
$$

holds for all $f \in \mathfrak{M}^{+}(0,+\infty)$ since its left part is equal 0 . However, the inequality

$$
\left(\int_{0}^{+\infty} f(y)^{-1} d y\right)^{-1} \leq C\left(\int_{0}^{+\infty} u(x)\left(\int_{0}^{x} f(y) v(y) d y\right)^{-1} d x\right)^{-1}
$$

does not hold, for instance, for $f=v$.

Example 2. By Proposition 6 and the corollary if $v(y)=y^{3} \chi_{(0,+\infty)}(y)$, then the inequality

$$
\left(\int_{-1}^{+\infty}\left[g(y) v(y)^{-1}\right]^{\frac{1}{2}} d y\right)^{2} \leq C\left(\int_{-1}^{+\infty} \frac{1}{x^{2}}\left(\int_{-1}^{x} g(y) d y\right)^{\frac{1}{2}} d x\right)^{2}
$$

holds for all $g \in \mathfrak{M}^{+}(-1,+\infty)$ for some constant $C$, but the inequality

$$
\left(\int_{-1}^{+\infty} g(y)^{\frac{1}{2}} d y\right)^{2} \leq C\left(\int_{-1}^{+\infty} \frac{1}{x^{2}}\left(\int_{-1}^{x} g(y) v(y) d y\right)^{\frac{1}{2}} d x\right)^{2}
$$

does not hold, for instance, for $g=\chi_{(-1,0)}$. 
Proposition 1. Let $p, q \in(-\infty, 0)$ and $I=I^{\times}$or $I=I_{\times}$. Then the inequality

(13) $\left(\int_{a}^{b} f(x)^{p} d x\right)^{\frac{1}{p}} \leq C\left(\int_{a}^{b}\left[u(x)^{\frac{1}{q}}(I(f v))(x)\right]^{q} d x\right)^{\frac{1}{q}}$

for all $f \in \mathfrak{M}^{+}(a, b)$

holds, iff $u<+\infty$ a.e. on $(a, b),\left(\operatorname{Iv}^{p^{\prime}}\right)(t)>0$ for all $t \in(a, b)$ and (3) holds.

Proof: Let $E:=\{x \in(a, b) \mid u(x)=+\infty\}$.

Necessity. If mes $E>0$, then, for all measurable $f \geq 0$, we have

$$
\left(\int_{a}^{b}\left[u(x)^{\frac{1}{q}}(I(f v))(x)\right]^{q} d x\right)^{\frac{1}{q}} \leq\left(\int_{E}\left[u(x)^{\frac{1}{q}}(I(f v))(x)\right]^{q} d x\right)^{\frac{1}{q}}=0 .
$$

However, for $f=+\infty \chi_{(a, b)}$ the left part of (13) is greater than 0 . This contradiction implies the first condition. If $\left(I v^{p^{\prime}}\right)(t)=0$ for some $t \in$ $(a, b)$, then, for all measurable $f \geq 0$,

$$
\left(\int_{a}^{b}\left[u(x)^{\frac{1}{q}}(I(f v))(x)\right]^{q} d x\right)^{\frac{1}{q}} \leq\left[\left(I\left[u(x)^{\frac{1}{q}}(I(f v))(x)\right]^{q}\right)(t)\right]^{\frac{1}{q}}=0
$$

that is we have the second condition. The condition (3) follows directly from (13), since $\left[u(x)^{\frac{1}{q}}(I(f v))(x)\right]^{q} \geq u(x)[(I(f v))(x)]^{q}, x \in(a, b)$.

Sufficiency. Fix any measurable $f \geq 0$. If mes $\{x \in(a, b) \mid f(x)=$ $0\}>0$, then $\int_{a}^{b} f(x)^{p} d x=+\infty$ and (13) holds. Now let $f(x)>0$ for almost every $x \in(a, b)$. Then $(I(f v))(x)>0$ for all $x \in(a, b)$ (otherwise we have a contradiction with the second condition) and

$$
\left[u(x)^{\frac{1}{q}}(I(f v))(x)\right]^{q}=u(x)[(I(f v))(x)]^{q} \text { for all } x \notin E .
$$

Thus, (3) implies (13).

By applying Theorem 3, Proposition 1 and Theorems 1, 2 we find a precise characterization of $(3)$ for the range $p, q \in(0,1)$. Denote $\left(I^{\times}\right)^{*}:=I_{\times}$and $\left(I_{\times}\right)^{*}:=I^{\times}$. 
Corollary. Let $p, q \in(0,1), \frac{1}{r}:=\frac{1}{q}-\frac{1}{p}$ and $I=I^{\times}$or $I=I_{\times}$. Then $(a)$ of Theorem 1 is equivalent to

$\left(a_{3}\right) v>0$ a.e. on $(a, b),\left(I^{*} u\right)(t)>0$ for all $t \in(a, b)$ and $\mathcal{D}<+\infty$, where

$$
\mathcal{D}:= \begin{cases}\sup _{t \in(a, b)}\left[\left(I^{*} v^{p^{\prime}}\right)(t)\right]^{-\frac{1}{p^{\prime}}}\left[\left(I^{*} u\right)(t)\right]^{-\frac{1}{q}}, & p \geq q, \\ \left(\int_{a}^{b}\left[\left(I^{*} v^{p^{\prime}}\right)(t)\right]^{-\frac{r}{q^{\prime}}}\left[\left(I^{*} u\right)(t)\right]^{-\frac{r}{q}} v(t)^{p^{\prime}} d t\right)^{-\frac{1}{r}}, & p<q .\end{cases}
$$

Moreover, $\mathcal{D} \approx C$ for the least possible constant $C$ in $(3)$.

Proposition 2. Let $p, q \in(-\infty, 0)$ and $I=I^{\times}$or $I=I_{\times}$. Then the inequality (3) is equivalent to

$$
\begin{array}{r}
\left(\int_{a}^{b} g(y)^{p} v(y)^{-p} d y\right)^{\frac{1}{p}} \leq C\left(\int_{a}^{b} u(x)[(I g)(x)]^{q} d x\right)^{\frac{1}{q}} \\
\text { for all } g \in \mathfrak{M}^{+}(a, b) .
\end{array}
$$

Proof: Let $I=I^{\times}$and $b^{*}$ be defined as in Theorem 1 .

Let $(3)$ hold. Fix any measurable function $g \geq 0$. If there exists $x \in\left(b^{*}, b\right)$ such that $\int_{a}^{x} g<+\infty$, then

$$
\int_{a}^{x} g(y)^{p} v(y)^{-p} d y \geq\left(\int_{a}^{x} g\right)^{p}\left(\int_{a}^{x} v^{p^{\prime}}\right)^{1-p}=+\infty
$$

by Hölder's inequality with exponents $\frac{1}{p}$ and $\frac{1}{1-p}$. Hence, $\int_{a}^{b} g(y)^{p} v(y)^{-p} d y=+\infty$ and (14) holds.

Now let $\int_{a}^{x} g=+\infty$ for all $x \in\left(b^{*}, b\right)$. Put

$$
f(x):=+\infty \chi_{\left(b^{*}, b\right)}(x)+\left(g(x)^{p} v(x)^{-p}\right)^{\frac{1}{p}} \chi_{\left(a, b^{*}\right)}(x) .
$$

Then $f(x) v(x) \leq g(x)$ for any $x \in\left(a, b^{*}\right)$ such that $v(x)<+\infty$. This follows directly if $v(x)=0$ and this follows from the equality $\left(g(x)^{p} v(x)^{-p}\right)^{\frac{1}{p}}=$ $g(x) v(x)^{-1}$ in the case $v(x)>0$. Thus, $f v \leq g$ a.e. on $\left(a, b^{*}\right)$ by the 
definition of $b^{*}$. Therefore, we have

$$
\begin{aligned}
\left(\int_{a}^{b} g(y)^{p} v(y)^{-p} d y\right)^{\frac{1}{p}} & \leq\left(\int_{a}^{b^{*}} g(y)^{p} v(y)^{-p} d y\right)^{\frac{1}{p}} \\
& =\left(\int_{a}^{b} f(y)^{p} d y\right)^{\frac{1}{p}} \\
& \leq C\left(\int_{a}^{b} u(x)[(I(f v))(x)]^{q} d x\right)^{\frac{1}{q}} \\
& \leq C\left(\int_{a}^{b^{*}} u(x)[(I(f v))(x)]^{q} d x\right)^{\frac{1}{q}} \\
& \leq C\left(\int_{a}^{b^{*}} u(x)[(I g)(x)]^{q} d x\right)^{\frac{1}{q}} \\
& =C\left(\int_{a}^{b} u(x)[(I g)(x)]^{q} d x\right)^{\frac{1}{q}}
\end{aligned}
$$

Conversely, now let (14) hold. Fix any measurable function $f \geq 0$. If $\int_{a}^{b} f(x)^{p} d x=+\infty$, then (3) holds. Let $\int_{a}^{b} f(x)^{p} d x<+\infty$. Then

$$
\int_{a}^{x} f(y) v(y) d y \geq\left(\int_{a}^{x} f(y)^{p} d y\right)^{\frac{1}{p}}\left(\int_{a}^{x} v(y)^{p^{\prime}} d y\right)^{\frac{1}{p^{\prime}}}=+\infty
$$

for any $x \in\left(b^{*}, b\right)$. Put

$$
g(x):=+\infty \chi_{\left(b^{*}, b\right)}(x)+f(x) v(x) \chi_{\left(a, b^{*}\right)}(x) .
$$

Then $g(x)^{p} v(x)^{-p} \leq f(x)^{p}$ for any $x \in\left(a, b^{*}\right)$ such that $v(x)<+\infty$. This follows directly if $v(x)=0$ and this follows from the equality $g(x)^{p}=$ $f(x)^{p} v(x)^{p}$ in the case $v(x)>0$. Hence, $g(x)^{p} v(x)^{-p} \leq f(x)^{p}$ for almost 
every $x \in\left(a, b^{*}\right)$ by the definition of $b^{*}$. Therefore, we have

$$
\begin{aligned}
\left(\int_{a}^{b} f(x)^{p} d x\right)^{\frac{1}{p}} & \leq\left(\int_{a}^{b^{*}} f(x)^{p} d x\right)^{\frac{1}{p}} \\
& \leq\left(\int_{a}^{b^{*}} g(x)^{p} v(x)^{-p} d x\right)^{\frac{1}{p}} \\
& =\left(\int_{a}^{b} g(x)^{p} v(x)^{-p} d x\right)^{\frac{1}{p}} \\
& \leq C\left(\int_{a}^{b^{*}} u(x)[(I g)(x)]^{q} d x\right)^{\frac{1}{q}} \\
& =C\left(\int_{a}^{b^{*}} u(x)[(I(f v))(x)]^{q} d x\right)^{\frac{1}{q}} \\
& =C\left(\int_{a}^{b} u(x)[(I(f v))(x)]^{q} d x\right)^{\frac{1}{q}} .
\end{aligned}
$$

The proof of the case $I=I^{\times}$is complete and the case $I=I_{\times}$can be proved analogously.

Proposition 3. Let $p, q \in(-\infty, 0)$ and $I=I^{\times}$or $I=I_{\times}$. Then the inequality (13) is equivalent to

(15) $\left(\int_{a}^{b} g(y)^{p} v(y)^{-p} d y\right)^{\frac{1}{p}} \leq C\left(\int_{a}^{b}\left[u(x)^{\frac{1}{q}}(\operatorname{Ig})(x)\right]^{q} d x\right)^{\frac{1}{q}}$

$$
\text { for all } g \in \mathfrak{M}^{+}(a, b) \text {. }
$$

Proof: This proposition can be proved in the same way as the proof of Proposition 2. We only note that both (15) and (13) implies $u<+\infty$ a.e. on $(a, b)$ (see the proof of Proposition 1) and, consequently, for almost all $x \in(a, b)$ the equality $(I h)(x)=+\infty$ implies $u(x)^{\frac{1}{q}}(I h)(x)=+\infty$. 
Proposition 4. Let $p, q \in(-\infty, 0)$ and $I=I^{\times}$or $I=I_{\times}$. Then the inequality

$$
\begin{array}{r}
\left(\int_{a}^{b}\left[g(y) v(y)^{-1}\right]^{p} d y\right)^{\frac{1}{p}} \leq C\left(\int_{a}^{b} u(x)[(I g)(x)]^{q} d x\right)^{\frac{1}{q}} \\
\text { for all } g \in \mathfrak{M}^{+}(a, b)
\end{array}
$$

holds, iff $\operatorname{mes}\{x \in(a, b) \mid v(x)=+\infty\}>0$ or (3) holds.

Proof: Sufficiency. Let (3) hold. Fix an arbitrary measurable function $g \geq 0$. Put $f(x):=g(x) v(x)^{-1}$ into (3). Then we have (16) since $f(x) v(x) \leq g(x)$. Let $\operatorname{mes}\{x \in(a, b) \mid v(x)=+\infty\}>0$. Then

$$
\int_{a}^{b}\left[g(y) v(y)^{-1}\right]^{p} d y \geq \int_{\{x \in(a, b) \mid v(x)=+\infty\}}\left[g(y) v(y)^{-1}\right]^{p} d y=+\infty
$$

for any measurable function $g \geq 0$. Hence, the left part of (16) is zero.

Necessity. Let $\operatorname{mes}\{x \in(a, b) \mid v(x)=+\infty\}=0$ and (16) holds. Fix an arbitrary measurable function $f \geq 0$ and let the function $f_{0}$ be integrable on $(a, b)$ and $f_{0}(x) \in(0,+\infty), x \in(a, b)$. Denote $E:=\{x \in$ $(a, b) \mid v(x)=0\}$ and $E^{c}:=(a, b) \backslash E$. Put $g_{n}=f v \chi_{E^{c}}+\frac{1}{n} f_{0} \chi_{E}, n \in \mathbb{N}$ into (16). Then we obtain

$$
\begin{aligned}
\left(\int_{a}^{b} f(x)^{p} d x\right)^{\frac{1}{p}} & \leq\left(\int_{E^{c}} f(x)^{p} d x\right)^{\frac{1}{p}} \\
& =\left(\int_{a}^{b}\left[g_{n}(x) v(x)^{-1}\right]^{p} d x\right)^{\frac{1}{p}} \\
& \leq C\left(\int_{a}^{b} u(x)\left[\left(\operatorname{Ig}_{n}\right)(x)\right]^{q} d x\right)^{\frac{1}{q}}
\end{aligned}
$$

for all $n \in \mathbb{N}$. Since $u(x)\left[\left(I g_{n}\right)(x)\right]^{q} \uparrow u(x)[(I(f v))(x)]^{q}$ as $n \rightarrow \infty$ for every $x \in(a, b)$ the Monotone Convergence Theorem implies (3). 
Proposition 5. Let $p, q \in(-\infty, 0)$ and $I=I^{\times}$or $I=I_{\times}$. Then the inequality

$$
\begin{array}{r}
\left(\int_{a}^{b}\left[g(y) v(y)^{-1}\right]^{p} d y\right)^{\frac{1}{p}} \leq C\left(\int_{a}^{b}\left[u(x)^{\frac{1}{q}}(I g)(x)\right]^{q} d x\right)^{\frac{1}{q}} \\
\text { for all } g \in \mathfrak{M}^{+}(a, b)
\end{array}
$$

holds, iff $\operatorname{mes}\{x \in(a, b) \mid v(x)=+\infty\}>0$ or (13) holds.

Proof: Proposition 5 can be proved in a completely similar way as Proposition 4 , so we leave out the details.

Remark. It is clear that if $p, q \in(0,1)$, then (14), (15), (16) and (17) are equivalent, and (3) is equivalent to (13).

Proposition 6. Let $p, q \in(0,1)$ and $I=I^{\times}$. Then (16) holds, iff $\int_{t}^{\mathfrak{b}^{*}} u=+\infty$ for all $t \in\left(a, \mathfrak{a}^{*}\right), \int_{\mathfrak{b}^{*}}^{b} v(x)^{-p} d x=0$ and

(18) $\left(\int_{\mathfrak{a}^{*}}^{\mathfrak{b}^{*}} f(x)^{p} d x\right)^{\frac{1}{p}} \leq C\left(\int_{\mathfrak{a}^{*}}^{\mathfrak{b}^{*}} u(x)\left[\int_{\mathfrak{a}^{*}}^{x} f v\right]^{q} d x\right)^{\frac{1}{q}}$

$$
\text { for all } f \in \mathfrak{M}^{+}\left(\mathfrak{a}^{*}, \mathfrak{b}^{*}\right)
$$

holds, where $\mathfrak{a}^{*}:=\inf \{t \in(a, b] \mid \operatorname{mes}\{x \in(t, b) \mid v(x)=0\}=0\}$ and $\mathfrak{b}^{*}:=\inf \left\{t \in\left[\mathfrak{a}^{*}, b\right] \mid \int_{t}^{b} u=0\right\}$.

Proof: Necessity. Fix any $t \in\left(a, \mathfrak{a}^{*}\right)$. There exists $\gamma \in\left(t, \mathfrak{a}^{*}\right)$ such that $\operatorname{mes}\{x \in(t, \gamma) \mid v(x)=0\}>0$. Put $g:=\chi_{(t, \gamma)}$ into (16). Then we obtain

$$
\begin{aligned}
+\infty & =\left(\int_{t}^{\gamma} v(x)^{-p} d x\right)^{\frac{1}{p}} \leq C\left(\int_{a}^{b} u(x)\left[\int_{a}^{x} g\right]^{q} d x\right)^{\frac{1}{q}} \\
& =C\left(\int_{t}^{\mathfrak{b}^{*}} u(x)\left[\int_{t}^{x} g\right]^{q} d x\right)^{\frac{1}{q}} \leq C(\gamma-t)\left(\int_{t}^{\mathfrak{b}^{*}} u\right)^{\frac{1}{q}},
\end{aligned}
$$

that is, $\int_{t}^{\mathfrak{b}^{*}} u=+\infty$. Insert $g:=\chi_{\left(\mathfrak{b}^{*}, b\right)}$ into (16) and we find that $\int_{\mathfrak{b}^{*}}^{b} v(x)^{-p} d x=0$. 
Now fix any measurable $f \geq 0$. If $\int_{\mathfrak{a}^{*}}^{\mathfrak{b}^{*}} u(x)\left[\int_{\mathfrak{a}^{*}}^{x} f v\right]^{q} d x=+\infty$, then (18) holds. Let $\int_{\mathfrak{a}^{*}}^{\mathfrak{b}^{*}} u(x)\left[\int_{\mathfrak{a}^{*}}^{x} f v\right]^{q} d x<+\infty$. Denote

$$
E:=\left\{x \in\left(\mathfrak{a}^{*}, \mathfrak{b}^{*}\right) \mid u(x)\left[\int_{\mathfrak{a}^{*}}^{x} f v\right]^{q}<+\infty\right\} .
$$

By the definition of $\mathfrak{b}^{*}$ for any $\xi \in\left(\mathfrak{a}^{*}, \mathfrak{b}^{*}\right)$ there exists $x \in\left(\xi, \mathfrak{b}^{*}\right) \cap E$ such that $u(x) \neq 0$. Then $\int_{\mathfrak{a}^{*}}^{x} f v<+\infty$. Since $\xi$ was taken arbitrary we have $f v<+\infty$ a.e. on $\left(\mathfrak{a}^{*}, \mathfrak{b}^{*}\right)$ and

$$
\operatorname{mes}\left\{x \in\left(\mathfrak{a}^{*}, \mathfrak{b}^{*}\right) \mid f(x) \neq 0, v(x)=+\infty\right\}=0 .
$$

This relation and $v>0$ a.e. on $\left(\mathfrak{a}^{*}, \mathfrak{b}^{*}\right)$ imply that $f(y) v(y) v(y)^{-1}=f(y)$ for almost every $y \in\left(\mathfrak{a}^{*}, \mathfrak{b}^{*}\right)$. It remains only to put $g:=f v \chi_{\left(\mathfrak{a}^{*}, \mathfrak{b}^{*}\right)}$ into (16) to obtain (18).

Sufficiency. Fix any measurable $g \geq 0$. Let $\int_{a}^{\mathfrak{a}^{*}} g=0$. Insert $f(x)=$ $g(x) v(x)^{-1}, x \in\left(\mathfrak{a}^{*}, \mathfrak{b}^{*}\right)$, into (18). Since $f v \leq g$ and $\int_{\mathfrak{b}^{*}}^{b} v(x)^{-p} d x=0$ we obtain

$$
\begin{aligned}
\left(\int_{a}^{b}\left[g(y) v(y)^{-1}\right]^{p} d y\right)^{\frac{1}{p}} & =\left(\int_{\mathfrak{a}^{*}}^{\mathfrak{b}^{*}}\left[g(y) v(y)^{-1}\right]^{p} d y\right)^{\frac{1}{p}} \\
& \leq C\left(\int_{\mathfrak{a}^{*}}^{\mathfrak{b}^{*}} u(x)\left[\int_{\mathfrak{a}^{*}}^{x} g\right]^{q} d x\right)^{\frac{1}{q}} \\
& =C\left(\int_{a}^{b} u(x)\left[\int_{a}^{x} g\right]^{q} d x\right)^{\frac{1}{q}} .
\end{aligned}
$$

If $\int_{a}^{\mathfrak{a}^{*}} g>0$, then there exists a number $t \in\left(a, \mathfrak{a}^{*}\right)$ such that $\int_{a}^{t} g>0$ and

$$
\left(\int_{a}^{b} u(x)\left[\int_{a}^{x} g\right]^{q} d x\right)^{\frac{1}{q}} \geq\left(\int_{t}^{\mathfrak{b}^{*}} u\right)^{\frac{1}{q}} \int_{a}^{t} g=+\infty
$$

by the first condition.

Finally, in a similar way we can prove the following statement: 
Proposition 7. Let $p, q \in(0,1)$ and $I=I_{\times}$. Then (16) holds, iff $\int_{\overline{\mathfrak{a}}}^{t} u=+\infty$ for all $t \in(\overline{\mathfrak{b}}, b), \int_{a}^{\overline{\mathfrak{a}}} v(x)^{-p} d x=0$ and

$$
\begin{aligned}
\left(\int_{\overline{\mathfrak{a}}}^{\overline{\mathfrak{b}}} f(x)^{p} d x\right)^{\frac{1}{p}} \leq C\left(\int_{\overline{\mathfrak{a}}}^{\overline{\mathfrak{b}}} u(x)\left[\int_{x}^{\overline{\mathfrak{b}}} f v\right]^{q} d x\right)^{\frac{1}{q}} \\
\text { for all } f \in \mathfrak{M}^{+}(\overline{\mathfrak{a}}, \overline{\mathfrak{b}})
\end{aligned}
$$

holds, where $\overline{\mathfrak{b}}:=\sup \{t \in[a, b) \mid \operatorname{mes}\{x \in(a, t) \mid v(x)=0\}=0\}$ and $\overline{\mathfrak{a}}:=\sup \left\{t \in[a, \overline{\mathfrak{b}}] \mid \int_{a}^{t} u=0\right\}$.

Acknowledgement. The author expresses his deep gratitude to Professor Lars-Erik Persson for drawing the author's attention to this problem and for fruitful discussions. He also thanks Professor Vladimir D. Stepanov for valuable comments and remarks.

\section{References}

[1] P. R. Beesack and H. P. Heinig, Hardy's inequalities with indices less than 1, Proc. Amer. Math. Soc. 83(3) (1981), 532-536.

[2] G. Bennett, Some elementary inequalities. III, Quart. J. Math. Oxford Ser. (2) 42(166) (1991), 149-174.

[3] J. S. Bradley, Hardy inequalities with mixed norms, Canad. Math. Bull. 21(4) (1978), 405-408.

[4] G. H. Hardy, J. E. Littlewood and G. Pólya, "Inequalities", Reprint of the 1952 edition, Cambridge Mathematical Library, Cambridge University Press, Cambridge, 1988.

[5] A. Kufner And L.-E. Persson, "Weighted inequalities of Hardy type", World Scientific Publishing Co., Inc., River Edge, NJ, 2003.

[6] V. G. MAZ'JA, "Sobolev spaces", Translated from the Russian by T. O. Shaposhnikova, Springer Series in Soviet Mathematics, Springer-Verlag, Berlin, 1985.

[7] B. Muckenhoupt, Hardy's inequality with weights, collection of articles honoring the completion by Antoni Zygmund of 50 years of scientific activity, I, Studia Math. 44 (1972), 31-38.

[8] B. OpIC And A. Kufner, "Hardy-type inequalities", Pitman Research Notes in Mathematics Series 219, Longman Scientific \& Technical, Harlow, 1990.

[9] G. Sinnamon, Weighted Hardy and Opial-type inequalities, $J$. Math. Anal. Appl. 160(2) (1991), 434-445. 
[10] G. Sinnamon and V. D. Stepanov, The weighted Hardy inequality: new proofs and the case $p=1$, J. London Math. Soc. (2) 54(1) (1996), 89-101.

[11] G. TAlenti, Osservazioni sopra una classe di disuguaglianze, Rend. Sem. Mat. Fis. Milano 39 (1969), 171-185.

Computing Centre FEB RAS

Tikhookeanskaya 153

Khabarovsk 680042

Russia

E-mail address: dmitry@sm.luth.se, prohorov@as.khb.ru

Primera versió rebuda el 19 de novembre de 2003, darrera versió rebuda el 9 de març de 2004. 
